\title{
Understanding the TCM Role of Liver in the Treatment of Cough
}

\author{
Tong Tong Zheng* \\ As-You-Wish Healthcare Institute, Taiwan
}

Received: 跚: November 27, 2018; Published: 制: December 18, 2018

*Corresponding author: Tong Tong Zheng, As-You-Wish Healthcare Institute, Taiwan

\begin{abstract}
The TCM role of Liver understood with the meridian route and functions shows that the insulting sequence plays a key to understanding the relationship between Liver and Lung in the effect treatment of cough. Liver qi stagnation is the most important and common pattern in clinic that deserves attention, which can be interpreted with scientific evidence in nerve systems. However, there remains a long way to go for the integration of TCM with the conventional medicine.
\end{abstract}

Keywords: Cough; Liver Qi Stagnation; Liver Meridian; Yin-Yang

\section{Mini Review}

Cough is the reflex action with protection that helps the patients clear the airway of foreign irritants or particles commonly seen in pneumonia, upper respiratory tract infection, laryngitis, tuberculosis, COPD (chronic obstructive pulmonary disease), bronchitis, and pulmonary. With the duration from three weeks to over eight weeks, cough can be categorized to be the acute, the subacute, and the chronic that can affect the quality of life to patients as long as it continues for over two weeks [1,2]. Although the stimulations on the immune system for the symptoms resulted from the infections have been verified effective with the acupuncture treatments in the recent scientific studies $[3,4]$, how to make acupuncture treatments most effectively requires an accurate diagnosis that follows Zang-Fu organs, meridian theories, Yin-Yang, and acupoint indications. Different from the Western medicine, symptoms based on the meridian theory, the Yin- Yang balance, Qi, body, and fluids Blood in the traditional Chinese medicine (TCM) are generally broader with the pattern identification. Acupuncturist strictly depend on the TCM patterns to make diagnosis rather than follow the pathological classifications of diseases by the western medicine [5].

\section{Glance at the Functions of the Liver Meridian}

The harmony of spirit, body, and mind is the representation of Yin-Yang balance, which not only sees the body as a miniature of the universe but as the whole system in diagnosis and treatment. The Yin-Yang balance in traditional Chinese medicine is the abstract but unique concept that servers as the guideline for explaining the causes of the diseases and the treatments for two thousand years and the key to the accurate diagnosis and successful treatment outcomes. A disease is viewed as the imbalanced Yin and Yang as in (Figure 1). A human body is supported by the three essential and vital treasures Jing, Qi, and Shen. Jing, essence, is classified as Ying and refers to material that seems to bear high similarity to genes. The serious issues aroused with the damages to Jing affect the physiological and psychological developments. Qi is classified as Yang that can maintain the body to function normally for fighting against external evil Qi and transport Blood to nourish the all the systems of the body [6,7]. On the basis of Qi, Blood, Essence, and fluids, Liver, Heart, Spleen, Lung, and Kidney are five Ying Zang organs that co-work by the meridian systems with the six Fu Yang organs Gall Bladder, Large Intestine, Stomach, Small Intestine, Sanjiao, and Bladder to connect all the systems of the body.

\begin{tabular}{|cc|}
\hline Yin & Yang \\
\hline & \\
Blood & Qi \\
Material & Function \\
Zang & Fu \\
Yin meridian & Yang meridian \\
\hline
\end{tabular}

Figure 1: Characteristics of Yin and Yang. 
The nature of Liver presented in dispersion and ascent affect the body to function normally [8]. Liver stores Blood and regulates the blood volume throughout the body for nourishment of the constituents. Whether or not the Qi can flow freely within the body to ensure the optimized distribution of fluids and blood is governed by Liver. On the other hand, the Liver qi can also affect the Spleen qi to ascend and the Stomach qi descend correctly, and the regulation of emotional activities closely associated with ejaculation, impotence, and menstruation [9]. Qi circulates in the traditional twelve meridians to connect the tissues and organs together, which plays a dominating role in the therapeutic outcomes that can regulate the normal body function and reflect pathology for diagnosis. Meridians are divided into three Hand Yin meridians, three Foot Yin meridians, three Hand Yang meridians, and three Foot Yang meridians as in (Table 1) [10]. The Liver meridian classified as Foot Jueyin originates at the lateral side of the big toe, meets with the Spleen meridian at SP6. Along the inner side of the leg it goes upwards to meet with SP12 and SP13, and then encircles the pubic region to connect with the Conception Vessel at CV2, CV3, and CV4. It continues upwards to enter Liver, connecting with Gallbladder. This meridian goes into the rib cage in Lung to meet with PC1 and ascends through the neck and the throat to the eye, and finally continues upwards to connect with the Governing Vessel at the vertex of the head [11].

Table 1: Twelve meridians and pathways.

\begin{tabular}{|c|c|c|}
\hline Meridians & Abbreviations & Pathways \\
\hline 3 Yin Meridians of the Hand & (LU, HT, PC) & Chest to Hand \\
\hline 3 Yang Meridians of the Hand & (LI, SI, SJ) & Hand to Face \\
\hline 3 Yang Meridians of the Foot & (ST, BL, GB) & Face to Foot \\
\hline 3 Yin Meridians of the Foot & (SP, KI, LV) & Foot to Chest \\
\hline
\end{tabular}

\section{Pathological View of Liver with Cough}

It is stated that "Five Zang organs and six Fu organs make people cough, in addition to Lung." in the Chapter 38 On cough of the volume Su Wen (Essential Questions) of the Huang-Di-NeiJing (Essential Questions of Yellow Emperor's Inner Classic). This warning suggests that a TCM practitioner and an acupuncturist must consider the relationship of the other organs in treating cough based on the Five Elements theory to gain the whole picture of the etiology. Liver in the Five Elements theory refers to Wood while Lung is Metal that governs Qi. The meridian route of Liver indicates that the Liver meridian passes Lung and the insulting sequence shows that Liver can invade Lung. When Liver fails to deliver free flow of Qi to descend Lung qi downwards, cough, dyspnea, or asthma occur. Blood regulation and dispersing stagnated Qi rely on the smooth flow of Liver qi $[12,13]$. Unfortunately, Liver qi stagnation, one of the pathological changes of Liver, is the extremely common TCM pattern in clinic which can imped the circulation of Qi as long as liver is depressed with the negative emotions like anger, worry, depression, and resentment, which can be presented with the breast swelling, frequent sighs, the tightness/ tension around the chest and the epigastric region, the feeling of a lump in the throat, or sometimes stabbing pain in the hypochondriac region $[14,15]$.
Scientific researches have shown that the depression occurs when the neurotransmitter signaling is dysregulated. The experimental result indicates the biochemical processing to transform macronutrients into molecules is closely related to Liver qi because the production of mitochondrial ATP of neurons is disrupted with the insufficient deliverer of molecules, which is the presentation of Liver qi stagnation [16]. In addition, the malfunction of autonomic nervous system to control lymphocytes and granulocytes can be viewed as Liver qi stagnation on account that the ratio of the two cells can viewed as Yin and Yang and be affected with the dispersing function of Liver [17]. On the other hand, the sympathetic nervous system controls the Natural Killer (NK)-cells while the parasympathetic nervous system is assumed to be closely connected to the release of cytotoxic substances $[18,19]$.

\section{Discussion}

The quotation "Qi is the commander of Blood; Blood acts as the mother of Qi" highlights the collaboration of Blood and Qi. Blood is seen as Yin while Qi can be classified as Yang, which may cause diseases when the imbalance of Yin-Yang occurs. Scientific evidence shows that the nerve system is closely associated with the biochemical changes. Blood is produced with food qi by the Spleen, circulating in the veins governed by Heart to nourish the organs and the nerve systems. In addition to food qi, Blood is also generated by the mother of Liver Kidney with the Generating sequence, indicating that Kidney stores prenatal Jing and produces marrow. Marrow can then generate bone marrow that is transferred to manufacture Blood. Qi circulates in the traditional twelve meridians as an energy to support life, interacting with Blood for the Zang-Fu organs to function normally in harmony $[20,21]$. The research of the connection with oxygen metabolism and Qi in the twelve meridians highlights that one may be short of breath and experience wheezing or coughing when blood oxygen cannot be at the normal level, presenting the high physiological and pathological similarities in functions and reactions between Qi and oxygen [22].

In other words, this suggested that oxygen, to some extent, is equivalent to Qi [23]. The Generating Sequence shows that Kidney is the mother of Liver, suggesting that Kidney's problems can affect its child Liver. The maximum oxygen is delivered when the normal value of hematocrit remains at 45\% [24]. The kidney produces Erythropoietin to promote the number of red blood cells for increasing the capacity of the blood to carry oxygen. The oxygen circulation of the Kidney determines the production of EPO with the oxygen pressure in the tissues $[25,26]$. With the relationship between Liver and Kidney, it is suggested in this paper that Qi may be viewed as blood oxygen and Kidney is the key to the production of Qi, which can play an anti-inflammatory role with Liver. Stagnated Liver qi can be dispersed as long as Kidney can function normally with the delivery of the healthy Qi to Liver. In other words, the inflammation caused by the infection can be reduced with oxygen transported with the blood to Lung.

\section{Conclusion}

Inductive logic thinking is the basis of the developments of TCM and acupuncture theories. Acupuncturists and TCM practitioners 
stick to the pattern identification based on theories of Yin-Yang and the Five Elements that act as the two key principles in practice for the accurate diagnosis and the expected outcomes. The integration of the western medicine and acupuncture/TCM has attracted much more attention, which suggests that the trend may be the optimal choice for the public healthcare system. However, how to link these two systems together needs more research, which shows that there is a long way to go.

\section{References}

1. Gibson P, Wang G, Mc Garvey L, Vertigan AE, Altman KW, et al. (2016) Treatment of Unexplained Chronic Cough: CHEST Guideline and Expert Panel Report. Chest 149(1): 27-44.

2. Chung KF, Pavord ID (2008) Prevalence, pathogenesis, and causes of chronic cough. Lancet 371(9621): 1364-1374.

3. Zhang RY, Wang D, Wu JP, Li XL, Li CX, et al. (2016) Randomized Controlled Clinical Trials for Acupuncture Treatment of Pneumoconiosis. Zhen Ci Yan Jiu 41(2): 163-168.

4. Xu BQ (1986) Experimental studies on acupuncture treatment of acute bacillary dysentery - the role of humoral immune mechanism. In: Zhang XT (Eds.), Researches on acupuncture-moxibustion and acupunctureanesthesia. Science Press, Beijing, China, pp. 573-578

5. Acupuncture and Moxibustion Therapies for Coughing.

6. Tong Zheng Hong (2018) Challenges in Learning and Understanding Traditional Chinese Medicine and Acupuncture. Open Acc J Comp \& Alt Med 1(1).

7. Tong Zheng Hong (2018) An Exploration of Acupuncture Treatment to Meniere's disease. Advancements in Bioequivalence \& Bioavailability $1(5)$.

8. Zhao L, Wang T, Jian D, Chen A, Li G (2018) Liver-stomach disharmony pattern: Theoretical basis, identification and treatment. Journal of Traditional Chinese Medical Sciences 5(1): 53-57.

9. Maciocia G (1989) The foundations of Chinese Medicine. Library of Congress Cataloging in Publication Data NY.

10. Jing Luo (Channels and Collaterals | Meridians and Sub-Meridians. Sacred Lotus Chinese Medicine).

11. Deadman P, Al Khafaji M, Baker KA (2012) Manual of ACUPUNCTURE England, Journal of Chinese Medicine Publications.

ISSN: 2574-1241

DOI: 10.26717/BJSTR.2018.12.002238

Tong Tong Zheng. Biomed J Sci \& Tech Res

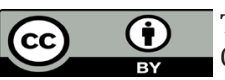

This work is licensed under Creative Commons Attribution 4.0 License

Submission Link: https://biomedres.us/submit-manuscript.php
12. Chu Q, Gong C (2014) Acupuncture for Dysmenorrhea, Internatioal Journal of Clinical Acupuncture 23(2): 67-73.

13. Mc Phee SJ, Papadakis MA (2012) Current Medical Diagnosis \& Treatment. Access Medicine.

14. (2007) World Health Organization, WHO International Standard Terminologies on Traditional Medicine in the Western Pacific Region, World Health Organization, Western Pacific Region.

15. Li J, Bi L, Xia K, Gao K, Chen J, et al. (2016) Biological basis of depression with liver-qi stagnation and spleen deficiency syndrome: A digital gene expression profiling study. Journal of Traditional Chinese Medical Sciences. 2(3): 150-158.

16. So R, Wong HS, Ko KM (2015) A Traditional Chinese Medicine Approach in Treating Depression by Promoting Liver Qi Circulation: A Western Medicine Perspective. Chinese Medicine 6(4): 187-195.

17. Abo T, Kawamura $\mathrm{T}$ (2002) Immunomodulation by the autonomic nervous system: therapeutic approach for cancer, collagen diseases, and inflammatory Bowel diseases. Ther Apheresis 6(5): 348-357.

18. Kawamura T, Toyabe S, Moroda T, Iiai T, Takahashi I, et al. (1997) Neonatal granulocytosis is a postpartum event which is seen in the liver as well as in the blood. Hepatology 26: 1567-1572.

19. Toyabe S, Iiai T, Fukuda M, Kawamura T, Suzuki S, et al. (1997) Identification of nicotinic acetylcholine receptors on lymphocytes in periphery as well as thymus in mice. Immunology 92(2): 201-205.

20. Tong Zheng Hong (2017) Exploring a New Extra Point for Sub-acute Cough: A Case Report. Scholar's Press, Germany.

21. Tong Zheng Hong (2017) Exploring a New Extra Point for Subacute Cough: A Case Report. Journal of Complement Medicine and Alternative Healthcare 3(4).

22. Liang Z, Huang B, Chen J (2012) Oxygen metabolism and meridian qi. Zhongguo Zhen Jiu 32(2): 183-186.

23. (2017) Lung Institute. Low Blood Oxygen and How it Affects the Body.

24. Donnelly S (2001) Why is erythropoietin made in the kidney? The kidney functions as a critmeter. Am J Kidney Dis 38(2): 415-425.

25. Donnelly S (2001) Why is erythropoietin made in the kidney? The kidney functions as a critmeter. Am J Kidney Dis 38(2): 415-425.

26. Siamak N Nabili, Erythropoietin (EPO, The EPO Test).

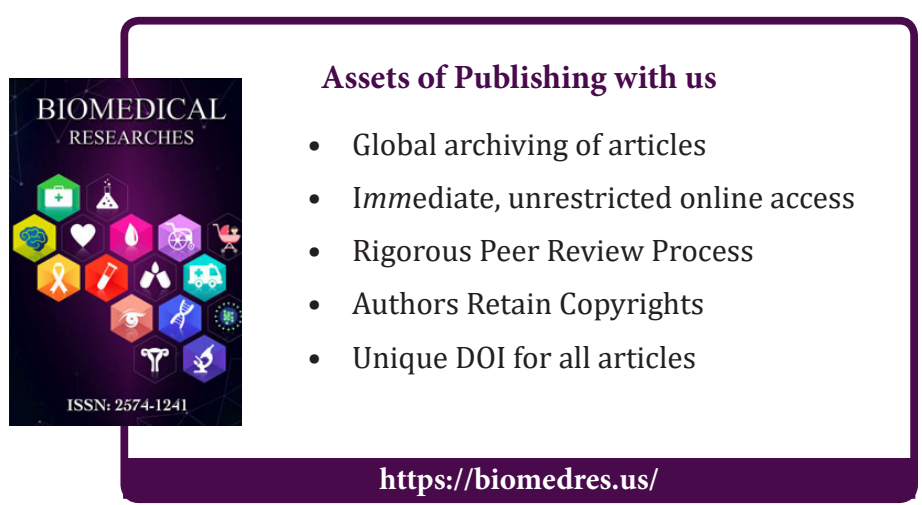

